\title{
Stereoselective binding of mexiletine and ketoprofen enantiomers with human serum albumin domains
}

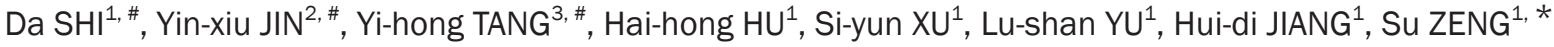 \\ ${ }^{1}$ Department of Drug Metabolism and Pharmaceutical Analysis, College of Pharmaceutical Sciences, Zhejiang University, Hangzhou \\ 310058, China; ${ }^{2}$ Taizhou University, Taizhou 317000, China; ${ }^{3}$ Shanghai Institute of Technology, Shanghai 201418, China
}

\begin{abstract}
Aim: To investigate the stereoselective binding of mexiletine or ketoprofen enantiomers with different recombinant domains of human serum albumin (HSA).

Methods: Three domains (HSA DOM I, II and III) were expressed in Pichia pastoris GS115 cells. Blue Sepharose 6 Fast Flow was employed to purify the recombinant HSA domains. The binding properties of the standard ligands, digitoxin, phenylbutazone and diazepam, and the chiral drugs to HSA domains were investigated using ultrafiltration. The concentrations of the standard ligands, ketoprofen and mexiletine were analyzed with HPLC.

Results: The recombinant HSA domains were highly purified as shown by SDS-PAGE and Western blotting analyses. The standard HSA ligands digitoxin, phenylbutazone and diazepam selectively binds to DOM I, DOM II and DOM III, respectively. For the chiral drugs, $R$-ketoprofen showed a higher binding affinity toward DOM III than S-ketoprofen, whereas S-mexiletine bound to DOM II with a greater affinity than $R$-mexiletine.
\end{abstract}

Conclusion: The results demonstrate that HSA DOM III possesses the chiral recognition ability for the ketoprofen enantiomers, whereas HSA DOM II possesses that for the mexiletine enantiomers.

Keywords: mexiletine; ketoprofen; human serum albumin; protein binding; stereoselectivity; ultrafiltration technique; Pichia pastoris GS115 cells

Acta Pharmacologica Sinica (2012) 33: 710-716; doi: 10.1038/aps.2012.8

\section{Introduction}

Human serum albumin (HSA) is the most abundant protein in human blood plasma, accounting for about half of the blood serum protein. After systemic absorption, most drugs undergo some degree of reversible binding to $\mathrm{HSA}^{[1]}$. The two enantiomers of a chiral drug may bind to HSA with different affinities, resulting in different free fractions. Over 50\% of the drugs in current clinical use are chiral, and the majority of synthetically derived chiral drugs are administered as mixtures of the constituent stereoisomers (most commonly the racemate $)^{[2]}$. In addition to stereoselective metabolism, stereoselective protein binding might also be responsible for the differences in pharmacokinetics between enantiomers ${ }^{[3-5]}$. Studies on the mechanism of stereoselective binding to HSA may better explain the different pharmacokinetics between enantiomers.

\footnotetext{
\#These three authors contributed equally to this work.

* To whom correspondence should be addressed.

E-mail zengsu@zju.edu.cn

Received 2011-08-10 Accepted 2012-01-15
}

HSA has a limited number of high-affinity binding sites for drugs $^{[6]}$. Fragments of HSA produced by chemical or enzymatic cleavage have been used to define the exact high-affinity binding sites for several ligands ${ }^{[7-10]}$. However, this method is limited by the finite number of cleavage sites in HSA, and chemical cleavage may destroy the structure of the binding site. Because HSA is composed of three quasi-independent domains, DOM I, DOM II, and DOM III ${ }^{[1]}$, a new method based on the cloning and expression of these three independent domains was first introduced by Dockal $\mathrm{M}^{[11]}$. Several studies have successfully employed recombinant HSA domains to identify the specific binding sites of several drugs, including warfarin, ochratoxin A, propofol and halothane ${ }^{[12-15]}$. The use of recombinant HSA fragments in a binding study with warfarin enantiomers demonstrated that the recombinant domains may also be a useful tool to reveal the stereoselective binding properties of chiral drugs ${ }^{[16]}$. In this study, we constructed three recombinant HSA domains to investigate the stereoselective binding properties of ketoprofen and mexiletine.

Ketoprofen and mexiletine are both chiral drugs and are currently used as racemates. The stereoselective binding of 
ketoprofen enantiomers to human serum albumin was discovered in $1980^{[17]}$. In 1990, Verbeeck et al determined that ketoprofen bound extensively to HSA (above 99\%) ${ }^{[18]}$, and that this binding may be related to the enantioselective disposition of ketoprofen in vivo. In the past several decades, studies on the stereoselective binding of ketoprofen to HSA have reached a consensus that ketoprofen mainly binds to site II of HSA and does so in a stereoselective manner ${ }^{[19-21]}$. However, contradictory stereoselective binding results have been obtained under different experimental conditions ${ }^{[17,18,22-24]}$. Studies using the method based on recombinant HSA domains may complement other binding studies to better understand the stereoselective binding properties of ketoprofen to HSA. Mexiletine is $70 \%$ bound to serum protein ${ }^{[25]}$, and the stereoselective disposition of mexiletine in man was first studied in $1986^{[26]}$. In vitro studies using serum protein from healthy subjects indicated that mexiletine bound to serum protein in a stereoselective manner ${ }^{[27]}$. Because serum protein is made up of HSA and other proteins such as a-acid glycoprotein, the mechanism of stereoselective binding between mexiletine and HSA needs to be further studied. However, little progress has been made in identifying either binding sites in HSA or the chiral binding mechanism. In this study, the stereoselective properties of the binding between chiral drugs (mexiletine and ketoprofen) and HSA were investigated using purified recombinant HSA domains.

\section{Materials and methods Cloning}

This protocol was a modification of previously published methods ${ }^{[11,12]}$. In brief, the gene segments coding for the HSA domains (the domains contained the following amino acids: HSA DOM I, 1-197; HSA DOM II, 189-385; and HSA DOM III, 381-585) were amplified by polymerase chain reaction (PCR) using the pBS-HSA plasmid as the template. The forward and reverse primers (Table 1 ) were designed to incorporate EcoR I and Not I sites, respectively.

The PCR products were digested overnight and then ligated into the pPIC9 vector (Invitrogen), resulting in the recombinant vectors pPIC9-HSA DOM I, pPIC9-HSA DOM II, and

Table 1. Sequence of primers.

\begin{tabular}{ll}
\hline \multicolumn{1}{c}{ Primers } & \multicolumn{1}{c}{ Sequence } \\
Domain I & \\
Forward primer & ggcggaattcgatgcacacaagag \\
Reverse primer & atttgcggccgctctctgtttggc \\
Domain II & \\
Forward primer & agcagaattcgggaaggcttcgtct \\
Reverse primer & ataatgcggccgcctgaggctcttc \\
Domain III & \\
Forward primer & \\
Reverse primer & agacgaattcgtggaagagcctcag \\
\end{tabular}

pPIC9-HSA DOM III. The recombinant vectors were transformed into $E$ coli DH5a for amplification and subsequent DNA sequence analysis. The identified recombinant plasmids were linearized with Sal I and transformed into competent Pichia pastoris GS115 cells (Invitrogen). The transformants were screened for viability in the absence of glucose and histidine. The positive recombinants were confirmed by PCR and DNA sequencing.

\section{Expression and purification}

The recombinants were grown on YPD medium and then transferred to BMGY medium for induction with methanol. Methanol with a final concentration of $1 \%$ was added every 24 $\mathrm{h}$ to maintain induction. All incubations were performed at $28^{\circ} \mathrm{C}$ on an orbital shaker at $250 \mathrm{r} / \mathrm{min}$. Supernatant samples were collected every $12 \mathrm{~h}$ for SDS-PAGE and Western blot analysis.

The protein was purified using the modified procedure described by Matsushita $S^{[14]}$. All steps of the purification procedure were performed at $4{ }^{\circ} \mathrm{C}$. The supernatants were harvested at $72 \mathrm{~h}$ after induction, followed by filtration through a $0.45 \mu \mathrm{m}$ filter. Purification was performed by precipitation with $85 \%\left(\mathrm{NH}_{4}\right)_{2} \mathrm{SO}_{4}$. The resulting samples were passed through a preequilibrated Blue Sepharose column (Amersham). After washing with 50 volumes of buffer 1 (50 $\mathrm{mmol} / \mathrm{L} \mathrm{KH}_{2} \mathrm{PO}_{4}, \mathrm{pH} 7.0$ ), elution was performed with buffer $2\left(50 \mathrm{mmol} / \mathrm{L} \mathrm{KH} \mathrm{PO}_{4}, 1.5 \mathrm{~mol} / \mathrm{L} \mathrm{KCl}, \mathrm{pH} 7.0\right)$. The isolated protein samples were extensively dialyzed against Sorensen's phosphate buffer. The protein concentrations were measured by the Bradford method.

\section{Western blot analysis}

The purified protein samples were eluted by adding an equal volume of loading buffer and then running them on $12 \%$ SDSPAGE gels. The separated proteins were then transferred to PVDF membranes. The membranes were incubated in the presence of goat anti-human serum albumin polyclonal antibody (Beckman). Exposure to the primary antibody was followed by incubation with a horseradish peroxidase (HRP)conjugated secondary anti-goat IgG antibody (Sanying Biotechnology). The blots were developed using an enhanced chemiluminescence detection system (ECL) (Amersham) according to the manufacturer's instructions.

\section{Ultrafiltration}

The binding properties of the three recombinant domains with the standard ligands ketoprofen and mexiletine were investigated by ultrafiltration using a Microcon centrifugation system (America, Millipore) that utilized a filter membrane with a $30-\mathrm{kDa}$ cutoff at $37^{\circ} \mathrm{C}$. Phenylbutazone, diazepam and digitoxin, each of which binds to a specific site in HSA, were chosen as the standard ligands. Aliquots of $500 \mu \mathrm{L}$ of each HSA domain with standard ligands were centrifuged at 7000xg for $5 \mathrm{~min}$. For ketoprofen and mexiletine, the centrifugations were performed at 7500xg for $10 \mathrm{~min}$ and 10000xg for $15 \mathrm{~min}$, respectively. The ultrafiltrate $(150 \mu \mathrm{L})$ was collected 
and prepared as described above.

Nonspecific filter membrane binding was evaluated in protein-free phosphate-buffered saline (PBS). The mixture was transferred to the ultrafilter without incubation, followed by ultrafiltration at $2000 \mathrm{xg}$ for $5 \mathrm{~min}$ at $37^{\circ} \mathrm{C}$. The samples in PBS buffer with or without ultrafiltration were directly injected into an HPLC system.

The percentage adsorbed by the ultrafilter is calculated using the following formula:

$$
\mathrm{P} \%=1-A_{\text {ultrafiltrate }} / A_{\mathrm{PBS}}
$$

$A_{\text {ultrafiltrate, }}$ drug peak area in the ultrafiltrate; $A_{\mathrm{PBS}}$, drug peak area in PBS buffer.

\section{Sample preparation}

The ultrafiltrates for the standard ligands (phenylbutazone, diazepam and digitoxin) were directly injected into the HPLC system. For ketoprofen, $R$-flurbiprofen was used as the internal standard. Ketoprofen was activated with $1 \%$ triethylamine and $2 \%$ thionyl chloride (both in methylene chloride) and then reacted with $S$-(-)-1-(1-naphthyl) ethylamine (S-NEA) (Sigma) to generate diastereoisomeric amides ${ }^{[28]}$. For mexiletine, $R$-esmolol was used as the internal standard, and the chiral derivatization was performed at $35^{\circ} \mathrm{C}$ for $10 \mathrm{~min}$ with 2,3,4,6-tetra-O-acetyl- $\beta$-D-glucopyranosyl isothiocyanate (GITC) (Sigma) ${ }^{[29]}$.

\section{HPLC analysis}

The concentrations of the standard ligands, ketoprofen and mexiletine, were analyzed by HPLC. HPLC was performed on an Agilent 1100 system consisting of a G1311A pump, a G1315A (DAD) UV detector, a manual injector and ChemStation software. An Agilent Zorbax C18 (250 mm×4.6 mm, 5 $\mu \mathrm{m})$ column was used. An aliquot of $20 \mu \mathrm{L}$ of each sample was injected and analyzed at room temperature (Table 2).

\section{Results}

\section{Cloning expression and purification}

After induction with methanol, the supernatants of the pPIC9HSA DOM I, pPIC9-HSA DOM II, and pPIC9-HSA DOM III transformants were analyzed by SDS-PAGE and Western blot. A single band was present at approximately $23 \mathrm{kDa}$, as shown in Figure 1, in accordance with the previously reported molecular masses of 22860, 22519, and 23383 Da for of HSA DOM I, HSA DOM II, and HSA DOM III, respectively ${ }^{[11]}$. The recombinant domains were also identified by Western blot with an anti-human HSA antibody, as shown in Figure 2. The results

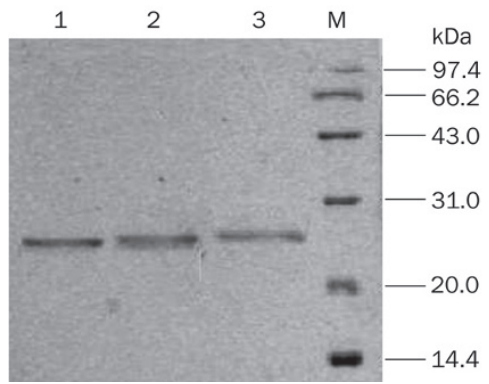

Figure 1. Analysis of the expression products by SDS-PAGE. (1) HSA DOM III; (2) HSA DOM II; (3) HSA DOM I. The positions of the molecular weight standards are indicated in the right-most lane.

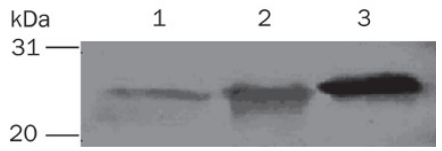

Figure 2. Analysis of the expression products by Western blot. (1) HSA DOM I; (2) HSA DOM II; (3) HSA DOM III. The positions of the molecular weight standards are indicated in the left-most lane.

confirmed that the recombinant HSA domains were successfully expressed and secreted into the supernatant.

To prevent the secreted foreign proteins from being degraded by the KEX-2 proteases present on the membrane of Pichia pastoris, tryptone was added to the culture, providing excess substrate for these proteases. Because higher concentrations of methanol might inhibit the expression of the target proteins, the final concentration of methanol was less than $1 \%$. As shown in Figure 3, the expression levels of the recombinant domains peaked at 72-96 h at a concentration of 210-275 $\mathrm{mg} / \mathrm{L}$. Consequently, the supernatants were harvested at $72 \mathrm{~h}$ for purification.

Although negligible levels of nonspecific proteins were detected by SDS-PSGE and Western blot, there were large amounts of mineral salts and metabolites in the supernatants. Impurities such as pigments, amino acids and carbohydrates may strongly inhibit the binding of the ligands with the recombinant protein fragments, resulting in a decreased protein binding rate. Blue Sepharose affinity chromatography was employed as the central step in the purification procedure. The Blue Sepharose column exhibited highly specific binding with the recombinant domains, as demonstrated by

Table 2. The estabolished HPLC methods.

\begin{tabular}{|c|c|c|c|}
\hline Drug & Mobile phase $(\mathrm{v} / \mathrm{v})$ & Flow rate $(\mathrm{mL} / \mathrm{min})$ & Detection $(\mathrm{nm})$ \\
\hline Phenylbutazone & Water-methanol (25:75) & 0.7 & 238 \\
\hline Diazepam & Water-methanol (25:75) & 0.7 & 242 \\
\hline Ketoprofen & Phosphate buffer (0.01 mol/L, pH 4.5)-acetonitrile (40:60) & 0.8 & 250 \\
\hline Mexiletine & Phosphate buffer (0.02 mol/L, pH 5.5)-acetonitrile (75:25) & 0.9 & 214 \\
\hline
\end{tabular}




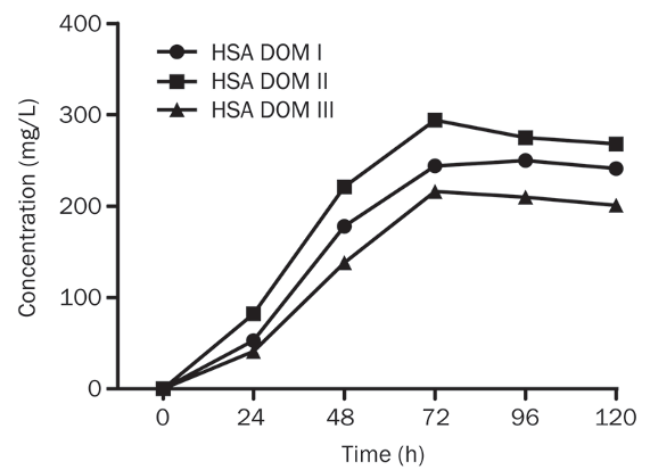

Figure 3. The protein expression in recombinant $P$ pastoris at different time points.

the fact that the purity of the domain preparation was greater than $98 \%$ in a previous study ${ }^{[11]}$. The purification efficiencies with or without precipitation were also compared. The recovery without precipitation is lower than that with precipitation (data not shown). These results indicated that the purification should be performed with precipitation. As shown in Figure 4 , the secreted protein segments were highly purified and concentrated after purification.

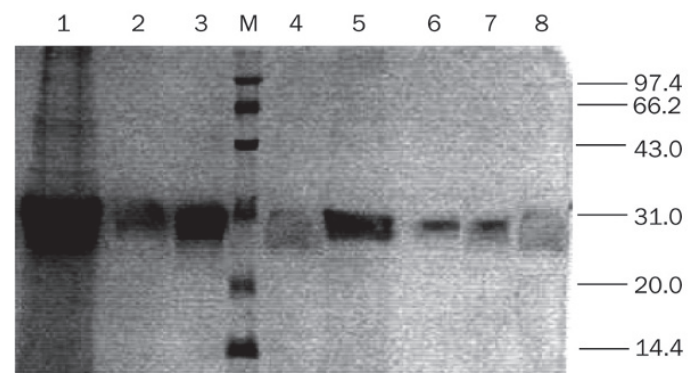

Figure 4. Analysis of the expression, precipitation and purification products by SDS-PAGE. (1) Precipitation of HSA DOM II; (2) Expression products of HSA DOM III; (3) Purification products of HSA DOM II; (4) Expression products of HSA DOM I; (5) Precipitation of HSA DOM I; (6) Purification products of HSA DOM I; (7) Purification products of HSA DOM III; (8) Expression products of HSA DOM III.

\section{Confirmation of nonspecific filter binding}

As shown in Table 3, the average nonspecific adsorption percentages under different concentrations were $3.53 \%$ for ketoprofen and $2.63 \%$ for mexiletine. These results indicated that the ultrafiltration system was suitable for studying the binding of ketoprofen and mexiletine to HSA.

Table 3. The nonspecific adsorption of ketoprofen and mexiletine with ultrafilter $(n=3)$.

\begin{tabular}{cccc}
\hline Drug & $\begin{array}{c}\text { Spiked amount } \\
(\mu \mathrm{g} / \mathrm{mL})\end{array}$ & $P(\%)$ & $P(\%)$ \\
\hline Ketoprofen & 1.0 & 3.77 & $3.53 \pm 0.50$ \\
& 5.0 & 3.86 & \\
Mexiletine & 50.0 & 2.95 & \\
& 0.5 & 2.25 & $2.63 \pm 1.57$ \\
& 5.0 & 1.28 & \\
\hline
\end{tabular}

\section{Binding with standard ligands}

It was widely accepted that the three recombinant domains possessed the three principal binding sites of HSA: the warfarin site (site I) in DOM II, the diazepam site (site II) in DOM III and the digitoxin site (site III) in DOM I ${ }^{[6]}$. In the present study, the binding properties of HSA and the three HSA domains with phenylbutazone, diazepam and digitoxin, each of which represents a standard ligand for HSA, were investigated. As shown in Figure 5, phenylbutazone bound to DOM II with greater affinity than to DOM I and showed no affinity to DOM III. Diazepam bound to DOM III with high selectivity. Digitoxin mainly bound to DOM I but also slightly bound to DOM II and DOM III. The results indicated that the primary binding sites of digitoxin, phenylbutazone and diazepam were on DOM I, DOM II, and DOM III, respectively. However, there may be low-affinity sites on DOM I for phenylbutazone and on DOM II and DOM III for digitoxin.

The binding abilities of the HSA domains were lower than
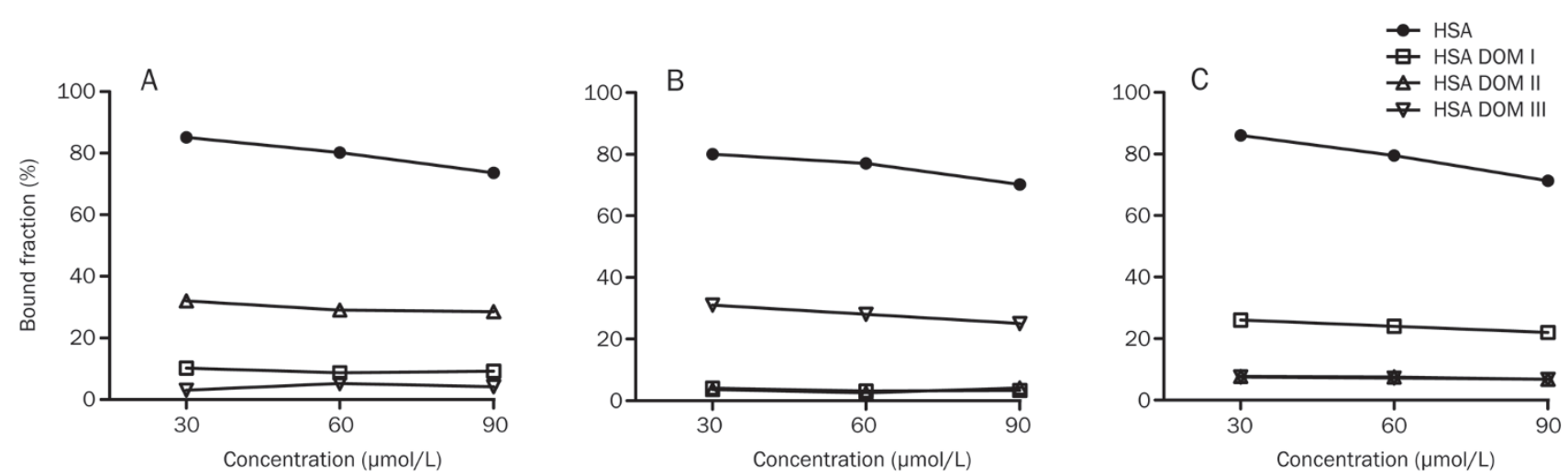

Figure 5. The protein binding of phenylbutazone (A), diazepam (B) and digitoxin (C) in $60 \mu \mathrm{mol} / \mathrm{L} \mathrm{HSA,} \mathrm{HSA} \mathrm{DOM} \mathrm{I,} \mathrm{HSA} \mathrm{DOM} \mathrm{II,} \mathrm{and} \mathrm{HSA} \mathrm{DOM} \mathrm{III.}$ 
those of rHSA, in agreement with the results of a previous report ${ }^{[14]}$ in which the site II marker DNSS bound with greater affinity to rHSA $(62.4 \% \pm 5.4 \%)$ than to domain III $(38.9 \% \pm 7.8 \%)$. The importance of the integrated three-dimensional structure may account for this phenomenon, as the interdomain interactions may maintain the stability of the ligand binding sites.

\section{Binding with the enantiomers of chiral drugs}

Contradictory results for the binding of ketoprofen enantiomers to HSA have been obtained in several studies ${ }^{[17,19,24,30]}$. Zou et al ${ }^{[24]}$ reported that the $S$-enantiomers bind to HSA more strongly than the R-enantiomers do. According to Dubois et $a l^{[19]}, R$-ketoprofen bound more strongly than S-ketoprofen, whereas Guo et al ${ }^{[31]}$ found that ketoprofen had little stereoselectivity with respect to binding to HSA. The binding of ketoprofen to DOM III showed remarkable stereoselectivity at the concentration of albumin found in plasma (5.2 $\mu \mathrm{mol} / \mathrm{mL})$, as $R$-ketoprofen exhibited a significantly higher binding affinity than $S$-ketoprofen $(P=0.0209)$. The bound fractions for $R$-ketoprofen and $S$-ketoprofen were $35.5 \% \pm 4.6 \%$ $(n=3)$ and $20.5 \% \pm 5.5 \%(n=3)$, respectively (Figure 6).

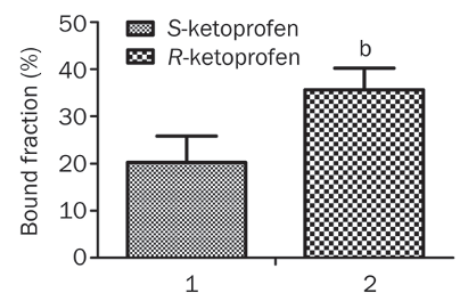

Figure 6. The binding of S-ketoprofen (1) and R-ketoprofen (2) (1.25 $\mu \mathrm{mol} / \mathrm{mL})$ to HSA DOM III $(5.2 \mu \mathrm{mol} / \mathrm{mL})(n=3)$.

For mexiletine, the primary binding site on HSA was site I in DOM II. Enantioselectivity was also observed for mexiletine enantiomers but was the opposite of that for ketoprofen. The binding of mexiletine enantiomers to HSA was significantly stereoselective, with the bound fractions of $S$-mexiletine and $R$-mexiletine being $44.35 \% \pm 1.9 \%(n=3)$ and $32.9 \% \pm 2.1 \%(n=3)$, respectively $(P=0.0022)^{[31]}$. The stereoselective trend was the same with the HSA domains. As shown in Figure 7, S-mexiletine [bound fraction of $55.3 \% \pm 14.6 \%(n=3)$ ] bound to DOM II

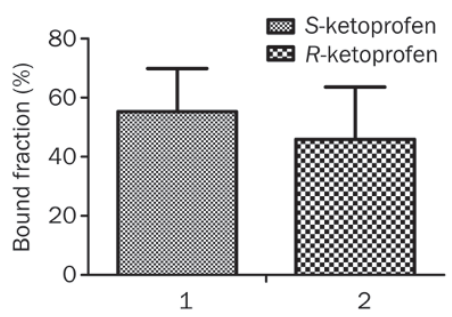

Figure 7. The binding of S-mexiletine (1) and R-mexiletine (2) (1.15 $\mu \mathrm{mol} / \mathrm{mL})$ to HSA DOM II $(4.9 \mu \mathrm{mol} / \mathrm{mL})(n=3)$. with a slightly greater affinity than $R$-mexiletine [bound fraction of $45.9 \% \pm 17.7 \%(n=3)]$. However, the stereoselectivity of the binding of mexiletine was not statistically significant, with a $P$ value of 0.3404 .

\section{Discussion}

\section{HSA binding with ketoprofen enantiomers}

Ketoprofen, a chiral non-steroidal anti-inflammatory drug (NSAID) of the 2-aryl propionate family, is currently marketed and used as a racemate. S-ketoprofen possesses most of the beneficial pharmacological activity ${ }^{[32]}$, whereas the $R$-enantiomer is considered to be an impurity or a pro-drug: approximately $10 \%$ of the $R$-enantiomer undergoes chiral inversion upon oral administration ${ }^{[33]}$. Ketoprofen bound extensively to HSA (above 99\%) ${ }^{[18]}$ in a stereoselective manner ${ }^{[34]}$, which may be related to the enantioselective disposition of ketoprofen in vivo ${ }^{[35,36]}$. Ketoprofen was reported to bind to HSA at site I and site II; the main binding site was site II (the high affinity binding site) in domain III $^{[19-21]}$. In this study, the bound fraction for ketoprofen racemate was in accordance with the results of the study by Matsushita ${ }^{[14]}$ (bound fraction of $64.0 \% \pm 5.4 \%$ for the ketoprofen racemate). These results indicated that the recombinant HSA domains produced in our study were highly purified and exhibited great activity.

The stereoselective HSA binding of ketoprofen has been identified by several studies ${ }^{[17,22-24,30]}$. However, contradictory results have been obtained under different experimental conditions. Dubois et $a l^{[19]}$ found that the enantioselective binding of ketoprofen enantiomers to HSA depended on drug and protein concentrations. Enantioselectivity was observed in HSA at $1 \mathrm{~g} / \mathrm{L}$, but the opposite enantioselectivity was observed at $40 \mathrm{~g} / \mathrm{L}$. At the concentration of HSA in plasma $(40 \mathrm{~g} / \mathrm{L})$, R-ketoprofen bound more strongly than the $S$-isomer, and the $k$ values of site II for $S$-ketoprofen were less than half of those for R-ketoprofen. Similar findings were obtained in our study, where the higher binding ability of $R$-ketoprofen to DOM III was detected at a similar physiological concentration. The method based on recombinant HSA domains may give a direct and thorough view of the stereoselective binding of chiral drugs.

\section{HSA binding with mexiletine enantiomers}

Mexiletine, an orally effective class 1 antiarrhythmic agent with a chiral center, is also used therapeutically as a racemate. The stereoselective disposition of mexiletine in humans was first revealed by Grech-Belanger et $a l^{[26]}$, who noted that the area under concentration-time curve (AUC) of $S$-mexiletine was always significantly higher $(P<0.01)$ and that the rate of renal clearance was significantly lower $(P<0.05)^{[3]}$ than that of the other enantiomer. It was reported that mexiletine was $70 \%$ bound to serum protein in healthy subjects ${ }^{[25]}$, and therefore, the differences observed between the pharmacokinetics of the enantiomers may be due largely to differences in their serum protein binding affinities.

As the stereoselective binding site and the mechanism of the binding of mexiletine enantiomers to HSA remain unknown, 
recombinant HSA domains were used in this study. This study represents the first attempt to identify the primary binding site of mexiletine to HSA, and it was determined that this binding site may be site I in DOM II. The bound fraction ratio of $S$-enantiomer to $R$-enantiomer on DOM II was 1.2 , a value that was in accordance with stereoselective binding to recombinant HSA (ratio is 1.34). The nonsignificant difference between $S$-mexiletine and $R$-mexiletine with respect to binding to DOM II may due to the large errors. Thus, more data should be gathered in future studies to confirm the stereoselectivity.

An in vitro binding study of mexiletine enantiomers further revealed that the serum binding of mexiletine could be accounted for primarily by binding to HSA and/or AGP $\mathrm{P}^{[25]}$. The binding of mexiletine enantiomers to AGP was examined in our previous study ${ }^{[31]}$, and the binding to AGP was also found to be stereoselective, with a bound fraction of $37.3 \%-24.1 \%$ for the $R$-enantiomer and a bound fraction of $31.1 \%-21.0 \%$ for the $S$-enantiomer. Although these results demonstrated opposite stereoselectivities for the binding to AGP and the binding to HSA, the stereoselective binding of the $S$-enantiomer to HSA may predominate in the plasma. Because the $S$-enantiomer possessed a slightly higher binding affinity for HSA DOM II, it had higher values for pharmacokinetic parameters, including the $\mathrm{AUC}^{[26]}$, the terminal elimination half-life ${ }^{[3]}$, and smaller values for parameters including renal clearance ${ }^{[26]}$ and steady-state volume of distribution ${ }^{[3]}$ than the $R$-enantiomer. These results confirmed the relationship between the HSA DOM binding of the mexiletine enantiomers and their pharmacokinetic properties.

\section{The application of recombinant HSA domains}

Recombinant HSA domains, first introduced in $1999^{[11]}$, have been used in several studies as powerful tools for ligand binding studies. To pinpoint the essential structural elements for the formation of the warfarin binding site on HSA, Dockal et al further constructed a defined set of five recombinant proteins ${ }^{[12]}$. Matsushita et $a l^{[14]}$ analyzed the function of three recombinant HSA domains and considered DOM I to be a potential protein carrier for drug delivery. The same recombinant HSA domains were employed by $\mathrm{Il}^{\prime}$ ichev et al ${ }^{[13]}$ to gain insight into the localization of binding sites and the nature of binding interactions between ochratoxin A and HSA. Liu et $a l^{[15]}$ utilized recombinant HSA domains to identify the main binding sites of two general anesthetics, propofol and halothane. Further analyses of the architecture of binding sites characterized the general anesthetic structure-activity relationship.

All of the above studies suggest that the recombinant HSA domains might be a suitable platform for the characterization of ligand binding. However, there has only been a single study applying the recombinant HSA domains to stereoselective binding research. Twine $e a l^{[16]}$ constructed two domain fragments of HSA corresponding to domains 1 and 2 (D12) and domains 2 and 3 (D23) and used these HSA fragments to study the binding of warfarin enantiomers to HSA. The results demonstrated that the fragments of HSA retained the ability to discriminate between pairs of warfarin enantiomers. In the present study, we performed stereoselective binding research using ketoprofen and mexiletine enantiomers and three recombinant HSA domains. This study complements other binding studies by revealing the binding properties of ketoprofen enantiomers to HSA. As the stereoselective binding of mexiletine to serum protein has not been well investigated, the nature of binding interactions between mexiletine enantiomers and HSA is further characterized in this study. The mexiletine binding sites are primarily found in DOM II, with an increased preference for the $S$-enantiomer.

In summary, we produced three highly purified recombinant HSA domains (HSA DOM I, HSA DOM II, and HSA DOM III), each of which had a specific ligand binding site. The recombinant domains were then employed to investigate the different chiral binding properties of the ketoprofen and mexiletine enantiomers. The results demonstrate that the method based on the recombinant HSA domains may have great potential to increase the understanding of the stereoselective binding properties of chiral drugs.

\section{Acknowledgements}

This project was supported by the State Key Development Program for Basic Research of China (Grant № 2011CB710800) and National Major Projects for Science and Technology Development of the Ministry of Science and Technology of China (№ 2012ZX09506001-004).

\section{Author contribution}

Su ZENG designed the study. Da SHI, Yin-xiu JIN, and Yihong TANG performed the experiments. Hai-hong HU and Si-yun XU contributed analytic tools. Lu-shan YU and Huidi JIANG analyzed the data. Da SHI, Yin-xiu JIN, Yi-hong TANG, and Su ZENG wrote the paper.

\section{References}

1 He XM, Carter DC. Atomic structure and chemistry of human serum albumin. Nature 1992; 358: 209-15.

2 Caner H, Groner E, Levy L, Agranat I. Trends in the development of chiral drugs. Drug Discov Today 2004; 9: 105-10.

3 Igwemezie L, Kerr C, McErlane K. The pharmacokinetics of the enantiomers of mexiletine in humans. Xenobiotica 1989; 19: 67782.

4 Hong $\mathrm{Y}$, Tang $\mathrm{Y}$, Zeng S. Enantioselective plasma protein binding of propafenone: mechanism, drug interaction, and species difference. Chirality 2009; 21: 692-8.

5 Sun DL, Huang SD, Wu PS, Li J, Ye YJ, Jiang HD. Stereoselective protein binding of tetrahydropalmatine enantiomers in human plasma, HSA, and AGP, but not in rat plasma. Chirality 2010; 22: 618-23.

6 Chuang VTG, Otagiri M. Stereoselective binding of human serum albumin. Chirality 2006; 18: 159-66.

7 Reed RG, Feldhoff RC, Clute O, Peters Jr T. Fragments of bovine serum albumin produced by limited proteolysis. Conformation and ligand binding. Biochemistry 1975; 14: 4578-83.

8 Bos OJM, Fischer MJE, Wilting J, Janssen LHM. Drug-binding and other physicochemical properties of a large tryptic and a large peptic 
fragment of human serum albumin. BBA-Protein Struct M 1988; 953 : 37-47.

9 Bos OJM, Remijn JPM, Fischer MJE, Wilting J, Janssen LHM. Location and characterization of the warfarin binding site of human serum albumin: A comparative study of two large fragments. Biochem Pharmacol 1988; 37: 3905-9.

10 Bos O, Labro J, Fischer M, Wilting J, Janssen L. The molecular mechanism of the neutral-to-base transition of human serum albumin. Acid/base titration and proton nuclear magnetic resonance studies on a large peptic and a large tryptic fragment of albumin. J Biol Chem 1989; 264: 953.

11 Dockal M, Carter DC, Ruker F. The three recombinant domains of human serum albumin. Structural characterization and ligand binding properties. J Biol Chem 1999; 274 : 29303-10.

12 Dockal M, Chang M, Carter DC, Rüker F. Five recombinant fragments of human serum albumin - tools for the characterization of the warfarin binding site. Protein Sci 2000; 9: 1455-65.

13 Il'ichev YV, Perry JL, Rüker F, Dockal M, Simon JD. Interaction of ochratoxin A with human serum albumin. Binding sites localized by competitive interactions with the native protein and its recombinant fragments. Chem-Biol Interact 2002; 141: 275-93.

14 Matsushita S, Isima Y, Chuang VTG, Watanabe H, Tanase S, Maruyama $\mathrm{T}$, et al. Functional analysis of recombinant human serum albumin domains for pharmaceutical applications. Pharm Res 2004; 21: 1924-32.

15 Liu R, Meng Q, Xi J, Yang J, Ha CE, Bhagavan NV, et al. Comparative binding character of two general anaesthetics for sites on human serum albumin. Biochem J 2004; 380: 147.

16 Twine S, Gore M, Morton P, Fish B, Lee A, East J. Mechanism of binding of warfarin enantiomers to recombinant domains of human albumin. Arch Biochem Biophys 2003; 414: 83-90.

17 Rendic S, Albic-Kolbah T, Kajfez F, Sunjic V. Stereoselective binding of $(+)$ and (-)-alpha-(benzoylphenylpropionic) acid (ketoprofen) to human serum albumin. Farmaco, Edizione Scientifica 1980; 35: 51-9.

18 Verbeeck R, Blackburn J, Loewen G. Clinical pharmacokinetics of nonsteroidal anti-inflammatory drugs. Clin Pharmacokinet 1983; 8: 297.

19 Dubois N, Lapicque F, Abiteboul M, Netter P. Stereoselective protein binding of ketoprofen: effect of albumin concentration and of the biological system. Chirality 1993; 5: 126-34.

20 Sakai T, Maruyama T, Imamura H, Shimada H, Otagiri M. Mechanism of stereoselective serum binding of ketoprofen after hemodialysis. J Pharmacol Exp Ther 1996; 278: 786.

21 Zhivkova ZD, Russeva VN. Stereoselective binding of ketoprofen enantiomers to human serum albumin studied by high-performance liquid affinity chromatography. J Chromatogr B 1998; 714: 277-83.

22 Hayball PJ, Nation RL, Bochner F, Newton JL, Massy-Westropp RA, Ha- mon DPG. Plasma protein binding of ketoprofen enantiomers in man: method development and its application. Chirality 1991; 3: 460-6.

23 Lapicque F, Muller N, Payan E, Dubois N, Netter P. Protein binding and stereoselectivity of nonsteroidal anti-inflammatory drugs. Clin Pharmacokinet 1993; 25: 115.

24 Zou $\mathrm{H}$, Wang $\mathrm{H}$, Zhang Y. Stereoselective binding of warfarin and ketoprofen to human serum albumin determined by microdialysis combined with HPLC. J Liq chromatogr R T 1998; 21: 2663-74.

25 Kwok D, Kerr C, McErlane K. Pharmacokinetics of mexiletine enantiomers in healthy human subjects. A study of the in vivo serum protein binding, salivary excretion and red blood cell distribution of the enantiomers. Xenobiotica 1995; 25: 1127-42.

26 Grech-Belanger O, Turgeon J, Gilbert M. Stereoselective disposition of mexiletine in man. Brit J Clin Pharmaco 1986; 21: 481.

27 McErlane K, Igwemezie L, Kerr C. Stereoselective serum protein binding of mexiletine enantiomers in man. Res Commun Chem Pathol Pharmacol 1987; 56: 141.

28 Jin YX, Tang YH, Zeng S. Analysis of flurbiprofen, ketoprofen and etodolac enantiomers by pre-column derivatization RP-HPLC and application to drug-protein binding in human plasma. J Pharmaceut Biomed 2008; 46: 953-8.

29 Jin YX, Zeng S. Determination of mexiletine enantiomers in human serum albumin after derivatization with GITC by RP-HPLC. Chinese Pharmaceutical Journal 2007. Chinese.

30 Guo CC, Tang YH, Hu HH, Yu LS, Jiang HD, Zeng S. Analysis of chiral non-steroidal anti-inflammatory drugs flurbiprofen, ketoprofen and etodolac binding with HSA. J Pharmaceut Anal; 1: 184-90.

31 Jin YX. Enantioselective binding of chiral drugs, mexiletine and ketoprofen, to plasma proteins. Thesis for master degree. Zhejiang University. 2006. p 24-31. Chinese.

32 Hutt A, Caldwell J. The importance of stereochemistry in the clinical pharmacokinetics of the 2-arylpropionic acid non-steroidal antiinflammatory drugs. Clin Pharmacokinet 1984; 9: 371-3.

33 Rudy AC, Liu Y, Brater C, Hall SD. Stereoselective pharmacokinetics and inversion of $(R)$-ketoprofen in healthy volunteers. J Clin Pharmacol 1998; 38: 3S.

34 Jamali F, Brocks D. Clinical pharmacokinetics of ketoprofen and its enantiomers. Clin Pharmacokinet 1990; 19: 197.

35 Grubb N, Rudy D, Brater D, Hall S. Stereoselective pharmacokinetics of ketoprofen and ketoprofen glucuronide in end-stage renal disease: evidence for a 'futile cycle' of elimination. Br J Clin Pharmacol 1999; 48: 494-500.

36 Bannwarth B, Lagrange F, Péhourcq F, Llanas B, Demarquez J. (S)ketoprofen accumulation in premature neonates with renal failure who were exposed to the racemate during pregnancy. $\mathrm{Br} J$ Clin Pharmacol 1999; 47: 459-60. 